Research Article

\title{
In-silico Recognition of Liquorice Phytoconstituents Specially Glabranin B as a Potential Cathepsin L Inhibitor to Hinder 2019-NCOV Host Cell Entry
}

\author{
Hoda Fathy ${ }^{1 *}$, Walid Abdelhady ${ }^{2}$, Reham S. Ibrahim ${ }^{1}$ \\ 1. Department of Pharmacognosy, Faculty of Pharmacy, Alexandria University, Alexandria, Egypt. \\ 2. Department of Clinical Pathology, Faculty of Medicine, Ainshams University, Cairo, Egypt. \\ *Corresponding author's E-mail: hodasherif@hotmail.com
}

Received: 19-06-2020; Revised: 24-08-2020; Accepted: 28-08-2020.

DOI: $10.47583 /$ ijpsrr.2020.v64i01.036

\begin{abstract}
The pandemic caused by novel coronavirus disease 2019 infecting millions of populations worldwide and the lack of specific treatment necessitate the use of all resources to remedy this scourge. The chemically-diverse natural products have been valuable sources for drug leads for centuries. Traditional Chinese Medicine (TCM) has a long history and achieved remarkable therapeutic effect during the treatment period of COVID-19 in China. This brief article discusses the therapeutic potential of some bioactive compounds from liquorice (Glycyrrhiza glabra) root for the treatment of COVID-19 by binding to cathepsin L. Virtual screening of a small database of 135 natural compounds from liquorice was applied via molecular docking using Schrodinger ${ }^{\circledR}$ Maestro interphase against cathepsin $L$ crystal structure; an endosomal cysteine protease necessary for coronavirus entry and replication. The structure activity relationship (SAR) of top hits was analyzed and the oral absorption and toxicity of the active compounds were predicted using Qikprop module. Based on the binding energy, Promising docking outcomes have been executed which evidenced the worthy of Liquorice as it contains several phytoconstituents acting as a potential inhibitor of cathepsin $L$ with the most active one the triterpenoidal saponin Glabrannin $B$ for future drug development to combat coronavirus disease and may help explaining the essential role of Liquorice in Chinese herbal medicine protocol for prevention and treatment of the coronavirus 19.
\end{abstract}

Keywords: COVID-19, liquorice, glabranin, cathepsin L, molecular docking.

\section{INTRODUCTION}

n December 2019, a novel Coronaviruses first outbreak in Wuhan, China named as severe acute respiratory syndrome coronavirus 2 (SARS-Cov-2) which have spread quickly worldwide, causing coronavirus disease-19 (COVID-19). As of 6 august, 2020, Globally, there have been 19,054,215 confirmed cases of COVID-19, including 712,619 deaths, reported to WHO. The clinical futures of COVID-19 mainly include fever, dry cough, tiredness and serious respiratory symptoms with high levels of morbidity and mortality.

A recent publication reported the structure of the Spike (S) protein of SARSCoV- 2 and showed that the ectodomain of its protein binds to the peptidase domain of angiotensinconverting enzyme 2 (ACE2). ${ }^{1}$ as the entry receptor and then uses the host serine protease TMPRSS2 for $\mathrm{S}$ priming, allowing fusion of viral and cellular membranes, hence viral entry into the cell. ${ }^{2}$ Cathepsin $L$ is a member of human Iysosomal cysteine proteases. ${ }^{3}$ SARS-CoV-2. enters the cell mainly through endocytosis, where cathepsin $L$ is critical for entry. ${ }^{4}$

Teicoplanin, an antibiotic significantly inhibits the invasion of cells by SARS-CoV via inhibiting the activity of cathepsin L. ${ }^{5}$ A recent study showed that the combined use of serine protease and cathepsin $\mathrm{L}$ inhibitors is effective block coronavirus host cell entry and intracellular replication. ${ }^{6}$

An in-vitro study confirmed that SARS-CoV infection and viral entry was blocked by specific inhibitors of cathepsin
L. SARS-CoV membrane fusion requires engagement of receptor followed by proteolysis cathepsin $L$ was sufficient to activate membrane fusion by SARS-CoV S as indicated by cell membrane fusion study ${ }^{7}$.

Traditional Chinese medicine (TCM) is based on more than 3,500 years of Chinese medical practice and includes herbal medicine, acupuncture and others. TCM has participated in the prevention and treatment of several epidemic diseases for example SARS epidemic in 2003 and a TCM scheme was included in the guideline on diagnosis and treatment during period of COVID-19 ${ }^{8}$.

Up to 60,107 of confirmed cases were treated by TCM in China. Ang et. al analyzed the composition of all the herbal formulae used in Chinese guide-line for COVID-19 in each disease stage and found that liquorice was the herb with the highest frequency of use regardless of disease stage. ${ }^{9}$ Chinese health authorities had issued programs recommending herbs for preventing COVID-19 infection with Liquorice on top of them ${ }^{10}$.

A study investigated the antiviral potential of glycyrrhizin in high doses, ribavirin and other compounds against two clinical isolates of coronavirus from patients with SARS found that the most potent inhibitor of SARS-COV replication in Vero cells was glycyrrhizin, with a selectivity index of $67^{11}$.

Glycyrrhizin, was found to be more potent than ribavirin, the most commonly used treatment for SARS. As it practically wipe out the SARS virus in infected monkey 
cells, according to virologist Jindrich Cinatl and his colleagues. As it makes it difficult for the SARS virus to attach to and invade a target cell. It also hinders virus reproduction, slowing its spread from one cell to the next. $^{12}$

These findings and others triggered us to investigate the effect of liquorice bioactive compounds as cathepsin L inhibitors via molecular docking to discover potential natural candidates that can hinder 2019-nCoV host cell entry. The structure activity relationship (SAR) of top hits was analyzed.In addition to their predicted In addition to their predicted oral absorption and toxicity profiles using Quikprop module.

\section{MATERIALS AND METHODS}

\section{Generation of 3D database of natural compounds}

An in-house database containing 135 naturally occurring compounds isolated from liquorice were imported as (.sdf) files from our previous work $^{13}$ in addition to The specific cathepsin L inhibitor (Z-FY-CHO) benzyl N-[(2S)-1-[[(2S)-1(4-hydroxyphenyl)-3-oxopropan-2-yl]amino]-1-oxo-3phenylpropan-2-yl]carbamate was retrieved from pubchem data. Their3D structures were drawn using Chemdraw software (Cambridge Soft Corporation, Cambridge, USA) and savedas (.mol) files. Then, these files were converted to (.sdf) file using jchem for Excel add-in (chemaxon ${ }^{\circledR}$ ) (Budapest,Hungary) to be ready for molecular docking

Generation of 3D database of drugs currently used for treatment of covid 19

Several compounds used at present for treatment or symptomatic treatment of covid 19 namely plaquenil, cobicistat, ribavirin, relacatib, ritonavir, Camostat, baricitinib, remdesivir, thalidomide, ambroxol, thalidomide, fingolimod, ruxolitinib, ciclesonide were suggested and retrieved from pubmed database of covid 19 as .sdf files.

\section{Ligands preparation}

Ligands were prepared using the LigPrep3.4 module of the Schrödinger. The ionization states of the ligands were generated at $\mathrm{pH}$ of 7 using Epik 2.3 and optimized by means of OPLS3 force field. The desalt option was activated and tautomers were generated for all ionization states at the selected $\mathrm{pH}$ range. Chiralities of the ligands, when present, were determined from the 3D structure and a maximum number of 32 stereoisomers were generated when chirality was not identified.

\section{Protein preparation}

The X-ray crystal structure of human cathepsin L (PDB: $5 \mathrm{MQY})$ in complex with 4-[1,3-benzodioxol-5-ylmethyl(2phenoxyethyl)amino]-5-fluoropyrimidine-2-carbonitrile was downloaded from RCBS Protein Data Bank (http://www.pdb.org), prepared, reviewed and modified using Schrödinger ${ }^{\circledR}$ software where hydrogens were added, water molecules beyond $5 \AA$ were deleted and the energy was minimized.

\section{Grid generation and GLID docking}

The docking grid was generated with the GLID grid generation module where boxes enclosing the centroids of co-crystallized ligands of the selected protein crystal structures were set as the grids.

Docking studies were performed using the extra precision (XP) docking protocol of GLIDE Schrödinger in Maestro interface to reveal the underlying modes of binding and interactions. For validation of the adopted docking protocol, redocking of the co-crystallized ligand was performed and the interacting residues in the active site were compared

\section{ADMET}

The active compounds were filtered according to their predicted oral absorption, distribution, metabolism, excretion and toxicity (ADMET) parameters calculated using Qikprop 3.5.

\section{RESULTS AND DISCUSSION}

\section{Molecular docking and interaction mechanisms of the most active compounds with cathepsin L}

The specific pocket of cathepsin $L$ in which the active hits were bound contained polar amino acids like Gln19, Asp162 and Hie163. These findings were in agreement with the previously published results ${ }^{14}$

The most active compound Glabranin B was bound through hydrogen bonding with Gln21, Asp 160 and Asp162 and through charged negative interactions with Glu159. Numerous hydrophobic interactions were observed with Cys22, Cys25, Leu69, Thr72, Ala135, Ala214, Ala215, and Phe143. It was also engaged with polar interactions with GIn21, Ser24, and Asn187. In addition to Salt bridging with Lys117 (Figure 1A). This strong inhibition advocates glabranin $B$ as a potential druggable anti-COVID19.

The second active hit; neoisoliquiritin interacted with numerous hydrogen bonds with the amino acids Asp162, Ala138 and Glu159 as well as polar interactions with Gln19, Ser158 and Ser213 and negatively charged interactions with Glu159 and Asp162. Hydrophobic interactions with Cys25, Ala135, Ala138 and Met161 also aided in the strong binding to cathepsin L (Figure 1B)

Analysis of binding modes of glucoliquiritin apioside; the third hit, revealed that besides the polar and non-polar amino acids contributions in the active site, it exhibited six H-bonding with Glu63, Ash71, Iys117, Glu159, Ser213 and Ala214 (Figure 1C).

The interacting amino acid residues of the co-crystallized ligand of the used cathepsin L crystalline structure (4-[1,3benzodioxol-5-ylmethyl(2-phenoxyethyl)amino]-5-

fluoropyrimidine-2-carbonitrile) were compared with those of the top hits. The common residues involved in the 
protein-ligand interaction confirmed that the successful docking procedure in the correct binding cavity.

It is noteworthy that these natural top-scored phytoconstituents surpassed in their binding free energies (Table 1 ) the selected drugs adopted from FDA drug repurposing criteria (figure 3). Only Chloroquine and cibosistat showed higher activity than the specific inhibitor $(-7.1,-6.2)$ it is worth mentioning that chloroquine is known to interfere with SARS-CoV-2 attempts to acidify the lysosomes and presumably inhibits cathepsins. ${ }^{15}$
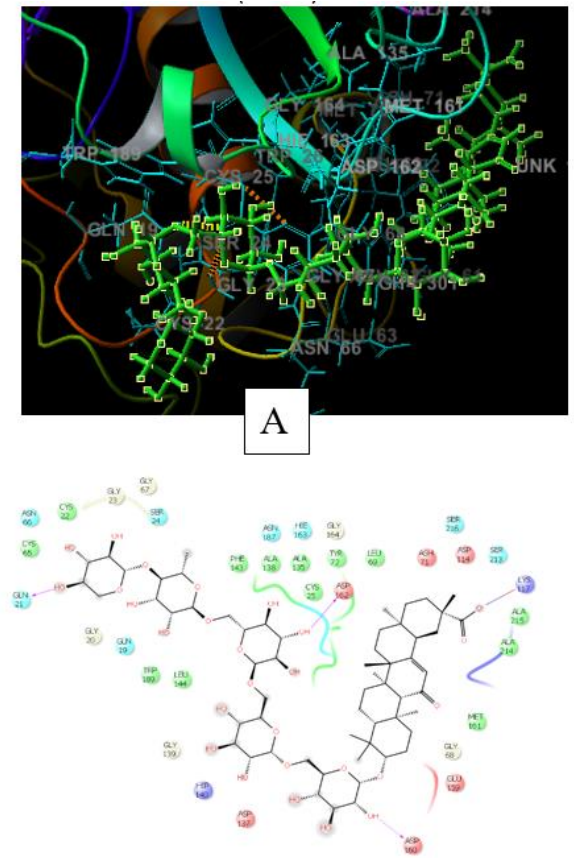
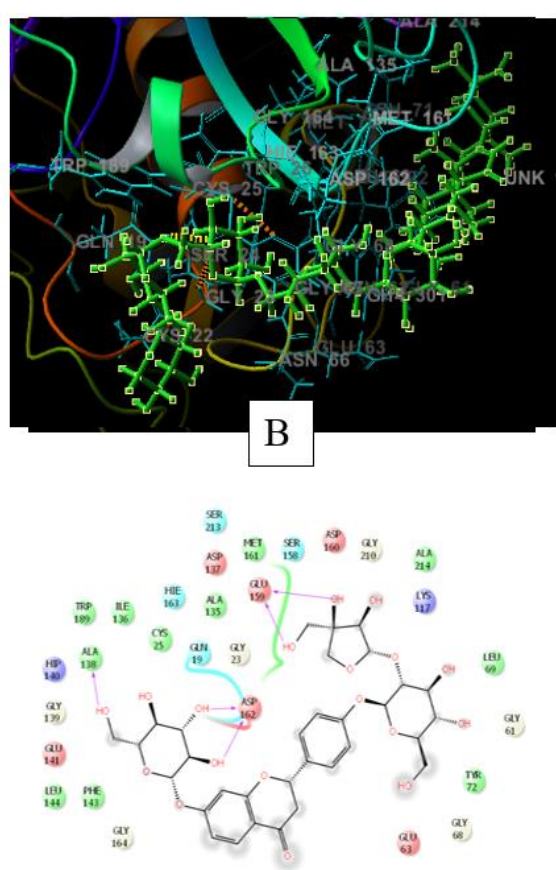
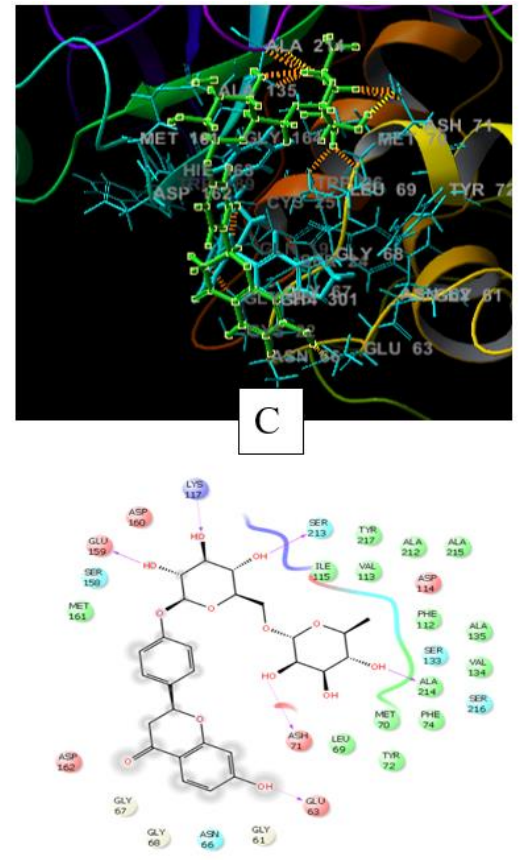

Figure 1: 2D and 3D interaction diagrams of $(A)$ glabranin $B,(B)$ neoisoliquiritine, $(C)$ glucoliquiritin apioside against cathepsin L crystal structure (5MQY)

It was interesting to observe that several potentially active natural compounds exerted lower docking scores than cathepsin L specific inhibitor (Table 1, figure 2).

Table 1: Chemical structures of the most active 50 liquorice compounds and the synthetic inhibitor against cathepsin $L$ and their XP G scores:

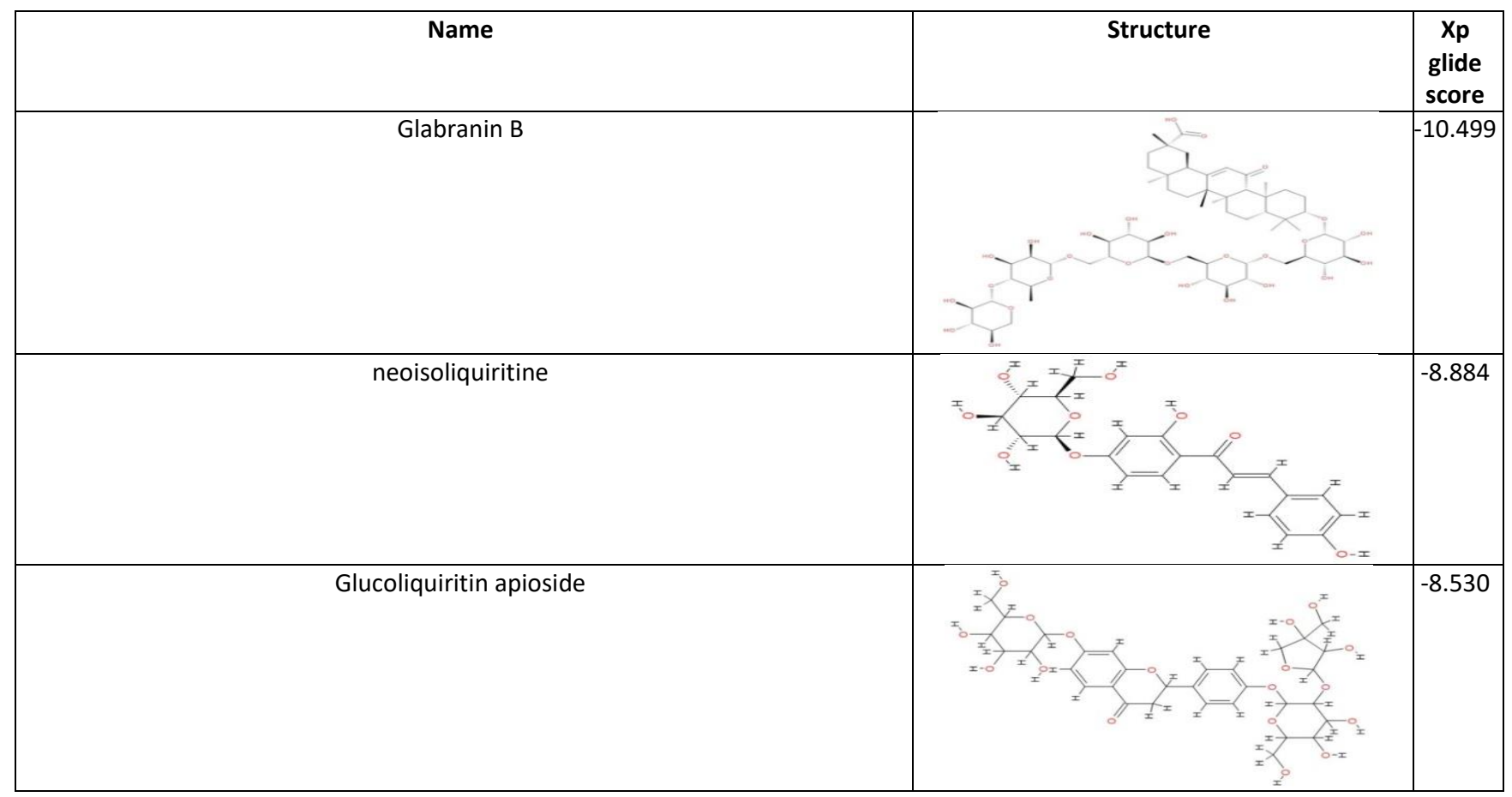




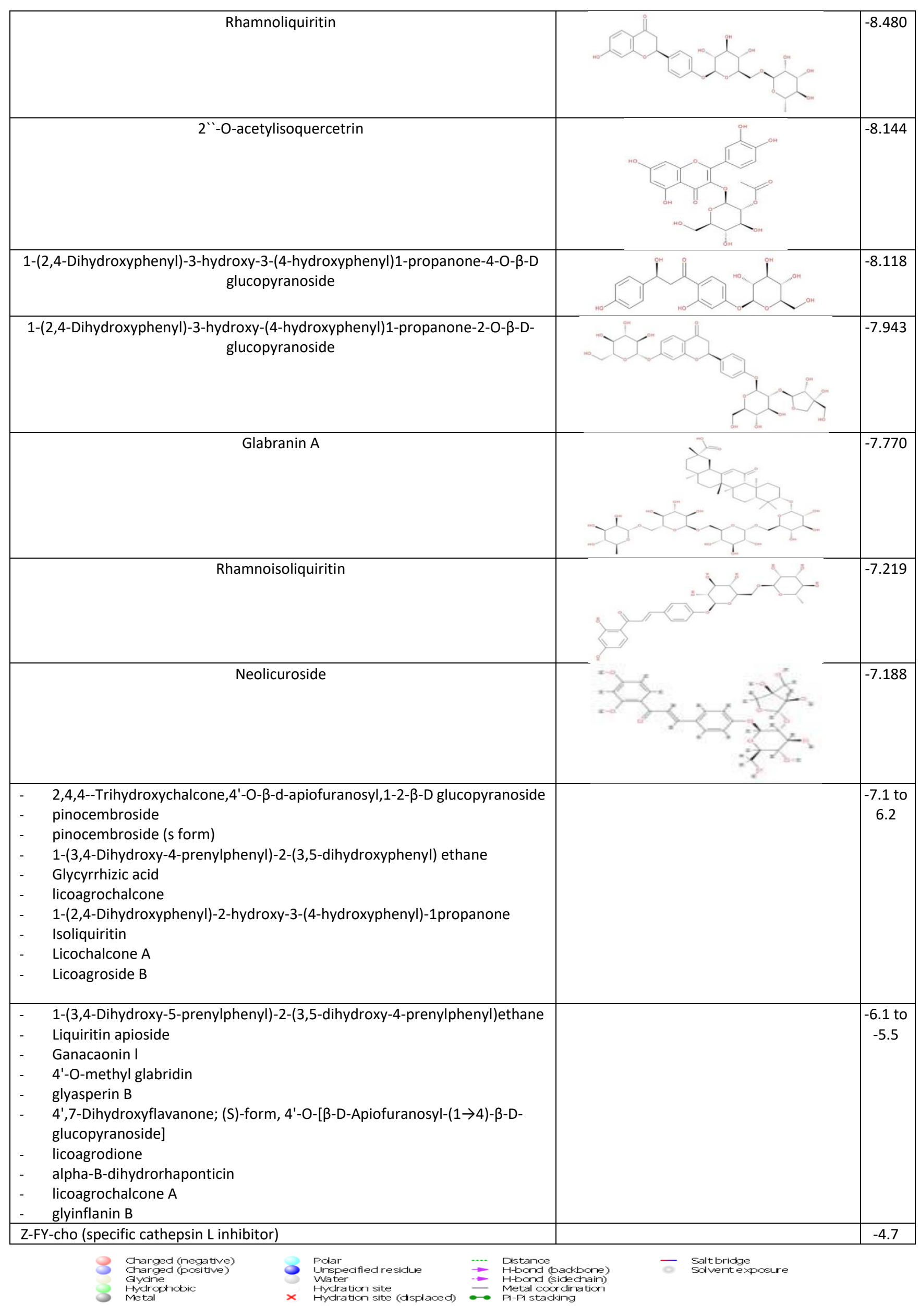




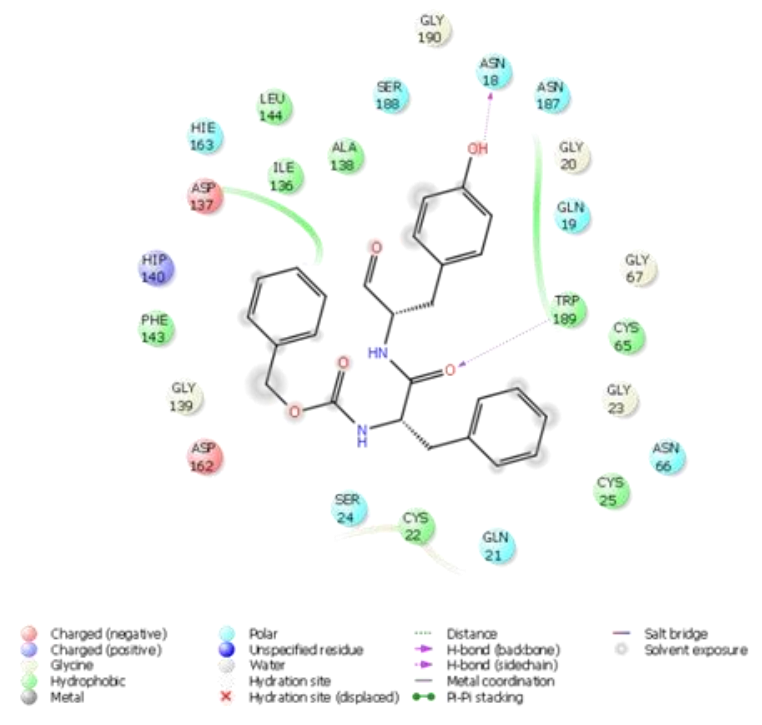

Figure 2. 2D interaction diagrams of Z-FY-cho against cathepsin L crystal structure (5MQY).

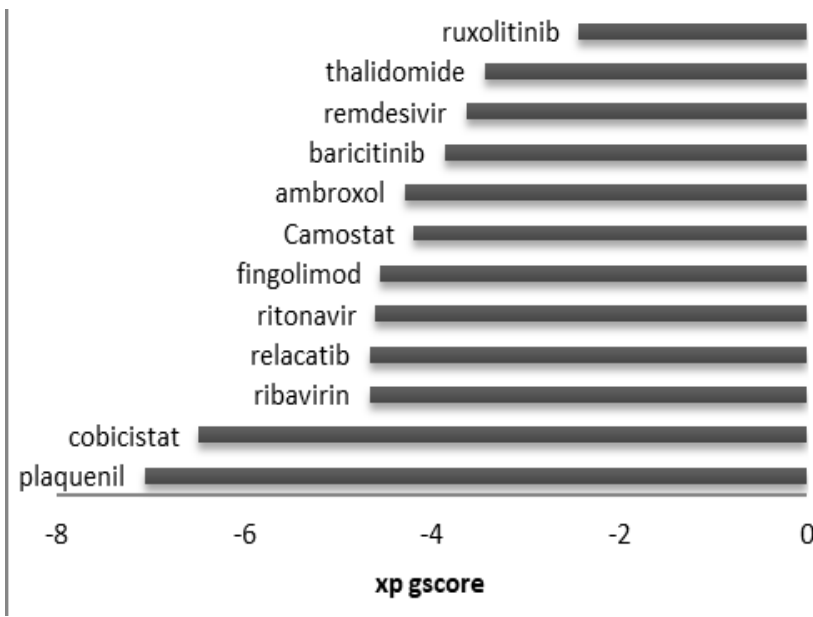

Figure 3: XP G-scores of FDA repurposed drugs currently used in the treatment of COVID-19.

\section{Structure activity relationship of the compounds:}

The top active 50 compounds were of diverse nature; mainly saponin, chalcone and flavanone. Glabranin B; a pentaglycoside of glycyrrhetic acid; the most active with the lowest XP G score (-10.499). The loss of one sugar unit in Glabranin A, decreased the activity $(-7.770)$ while the aglycone glycrrhizic acid showed even lower activity (6.644). Regarding chalcones, neoisoliquiritin; a 4monoglycoside; is the most active chalcone $(-8.884)$. The addition of sugar monomer or complete removal of sugar moieties greatly reduced the activity as shown in neolicuroside (-7.188), 2,4,4- Trihydroxychalcone 4'-0-8-dapiofuranosy-I,1 $\rightarrow 2-\beta-D$ glucopyranoside (-7.145), and rhamnoisoliquiritin (-7.219). Changing the position of glycosylation from 4 to 4 also reduced the activity as in isoliquiritin (-6.292) and licoagrochalcone (-6.559). The presence of $\mathrm{OH}$ group in position 1 seems to be essential for activity as that was evidenced by low activity of chalcones lacking it example 2',4',5,7-Tetrahydroxy-3',6diprenylisoflavan, 5-Methyl ether (-6.55).

\section{ADMET profiles of top 5 compounds}

Lipinski rule of 5 is a filter that help in early preclinical development and could help avoid costly late-stage preclinical and clinical failures. Although being questioned for stringency by Köster et al (Yu etal, 2012). It predicts if molecules comply with 2 or more of the following rules. a molecule with a molecular mass less than $500 \mathrm{Da}$, no more than 5 hydrogen bond donors, no more than 10 hydrogen bond acceptors, and an octanol-water partition coeffecient $\log P$ not greater than 5 . modified by Jorgensen rule of 3 , states that the aqueous solubility measured as logs should be $>-5.7$, the apparent Caco-2 cell permeability should be faster than $22 \mathrm{~nm} / \mathrm{s}$, and the number of primary metabolites should be $<7$.

Table 2: Expanded Lipinski's and Jorgensen's physicochemical parameter for top 5 active compounds against cathepsin L:

\begin{tabular}{|l|l|c|c|c|c|}
\hline ligand & $\begin{array}{l}\text { Molecular } \\
\text { weight }\end{array}$ & $\begin{array}{c}\text { Reactive } \\
\text { functional group } \\
\text { (rtvfg) }\end{array}$ & Oral absorption & Rule of five & Rule of three \\
\hline Glabranin B & 1235.375 & 5 & 1 & 3 & 2 \\
\hline Neoisoliquiritine & 714.098 & 2 & 2 & 1 & 1 \\
\hline Glucoliquiritin apioside & 712.657 & 3 & 1 & 3 & 2 \\
\hline Rhamnoliquiritin & 564.542 & 2 & 1 & 3 & 2 \\
\hline 2-O-Acetylisoquercetin & 684.38 & 2 & 1 & 3 & 2 \\
\hline
\end{tabular}

Molecules should preferably comply with 2 or more of the Lipinski and/ or Jorgensen rule. The maximum score of the top active 5 compounds was 3 for the rule of five and 2 for the rule of 3 (table 2). Glabranin $B$ is of molecular weight $>$ 1200 which may account for a poor bioavailability. It is a well known that glycyrrhizin the major triterpene saponin in Liquorice has less oral absorption than its sapogenin glycyrhetic acid In order to improve the bioavailability of glycyrrhizin, other nonvascular administration routes, as well as use of absorption enhancers, were studied. A rat study suggested that rectal or nasal administration of glycyrrhizin yielded 10 - or 26-times, respectively, improvement in the bioavailability of the licorice saponin as compared with p.o. administration ${ }^{14}$. In addition, several sodium fatty acids were found to be the absorption enhancers for glycyrrhizin. The same mechanisms or others could be used with glabranin B in case of clinical trials. It is also worth mentioning that glycyrhizic acid is also a potent cathepsin $\mathrm{L}$ inhibitor. 


\section{CONCLUSION}

The present study was carried to discover novel inhibitor molecules against cathepsin L, Our analyses revealed that glabrinin $B$ in addition to other top-scored phytoconstituents are natural cathepsin $L$ inhibitors from liquorice that might serve as druggable anti- SARS-CoV-2. We recommend further in vitro and in vivo studies for further innovation and development of antiviral compounds.

\section{REFERENCES}

1. Wrap D, Wang N, Corbett KS, Goldsmith J, Hsieh C, Abiona O, Graham B, Mclellan JS.. Cryo-em structure of the 2019ncov spike in the perfusion conformation. Science 13, mar: 2020, pp1260-1263.

2. Hoffmann $M$, Kleine-Weber $H$, Schroeder $S$, MÜller $M$, Drosten C, Pöhlmann SARS-CoV-2. Cell Entry Depends on ACE2 and TMPRSS2 and Is Blocked by a Clinically Proven Protease Inhibitor Cell 181(2), (2020), pp271-280.

3. Shah $P$, Wang $T$, Kaletsky R, Myers $M$, Purvis J, Jing $H$, Huryn D, Greenbaum D, Smith A, Bates P, and Diamond S. A smallmolecule oxocarbazate inhibitor of human cathepsin I blocks severe acute respiratory syndrome and ebola pseudotype virus infection into human embryonic kidney $293 \mathrm{~T}$ cells. Molecular Pharmacology vol. 78, no. 2, (2020), 319-324.

4. Ou, X., Liu, Y., Lei, X. et al. . Characterization of spike glycoprotein of SARS-CoV-2 on virus entry and its immune cross- reactivity with SARS-CoV. Nature Communications 11 , 2020, 1620. https://doi.org/10.1038/s41467-020-15562-9.

5. Baron S, Devaux C, Colson P, Raoult D, Rolain JM (2020). Teicoplanin: an alternative drug for the treatment of coronavirus COVID-19?, International Journal of Antimicrobial Agents, 55, 2020, 105944

doi: https://doi.org/10.1016/j.ijantimicag.2020.105944

6. Liu T, Luo S, Shi G Cathepsin L- selective inhibitor: A potentially promising treatment for covid-19 patients, Pharmacology and therapeutics 213, 2020, 107587

7. Simmons G, Gosalia D, Rennekamp A, Reeves J, Scott L. Diamond, and Paul Bates. Inhibitors of cathepsin L prevent severe acute respiratory syndrome coronavirus entry
Proceedings of the National Academy of Sciences August 16, 102 (33), 2005, pp11876-11881

8. Xu J, Zhang_Y. Traditional Chinese Medicine treatment of COVID-19, Complementary Therapies in Clinical Practice Volume 39, May, 2020, pp101165

9. Ang L, Lee H, Choi J, Zhang J, Lee M. (2020). Herbal medicine and pattern identification for treating COVID-19: a rapid review of guidelines, Integrative Medicine Research 9, 2020, pp100407.

10. Hui L, Qiao-ling T, Ya-xi S, Shi-bing L, Ming Y, Robinson N, Jianping L, Chin J. (2020) Can Chinese Medicine Be Used for Prevention of Corona Virus Disease 2019 (COVID-19)? A Review of Historical Classics, Research Evidence and Current Prevention. Journal of Integrative Medicine Apr 2020, 26(4), pp243-250.

11. Pilcher, H. Liquorice may tackle SARS. Nature. (2003) https://doi.org/10.1038/news030609-16

12. Cinatl J, Morgenstern B, Bauer G, Chandra P, Rabenau H, Doerr HW. Glycyrrhizin, an active component of liquorice roots, and replication of SARS-associated coronavirus. Lancet., 361(9374), 2003, pp 2045-2046. doi:10.1016/s0140-6736(03)13615-x

13. Ibrahim, R.S., Mahrous, R.S., Fathy, H.M. et al. Anticoagulant activity screening of an in-house database of natural compounds for discovering novel selective factor $\mathrm{Xa}$ inhibitors; a combined in silico and in vitro approach. Medicinal Chemistry Research 29, 2020, pp707726. https://doi.org/10.1007/s00044-020-02516-5

14. Raghav N, Kaur R. Chalcones, semicarbazones and pyrazolines as inhibitors of cathepsins B, $\mathrm{H}$ and $\mathrm{L}$. International Journal of Biological Macromoleule, 80, 2015 sep, pp $710-724$.

15. köster $\mathrm{H}$, Craan $\mathrm{T}$, Brass $\mathrm{S}$, Herhaus $\mathrm{C}$, Zentgraf $\mathrm{M}$, Neumann LA and Klebe G. A Small Nonrule of 3 Compatible Fragment Library Provides High Hit Rate of Endothiapepsin Crystal Structures with Various Fragment Chemotypes. Journal Medicinal Chemistry, 54, 22, 2011, pp7784-7796

16. Yu K, Chen F, and Li C Absorption, Disposition, and Pharmacokinetics of Saponins from Chinese Medicinal Herbs: What Do We Know and What Do We Need to Know More? Current Drug Metabolism, 13, 2012, pp 577-598.

Source of Support: None declared.

Conflict of Interest: None declared.

For any question relates to this article, please reach us at: editor@globalresearchonline.net

New manuscripts for publication can be submitted at: submit@globalresearchonline.net and submit_ijpsrr@rediffmail.com 